Conclusions Catheter ablation from the base of the NCC represents a safe and effective means to eliminate focal AT.

\section{e0557 CARTO MAPPING TO GUIDE ABLATION OF RIGHT VENTRICULAR OUTFLOW TRACT TACHYCARDIA VENTRICUALR CONTRACTION}

doi:10.1136/hrt.2010.208967.557

Zhou Xianhui, He Li, Tang Baopeng, Long Deyong, Li Jinxin, Zhang Yu, Xu Guojun, Zhang Jianghua. Pacing Electrophysiological Department, First Affiliated Hospital, Xinjiang Medical University

Objective The aim of this study was to determine whether CARTO mapping is feasible in the right ventricle and assess its utility in guiding ablation of right ventricular outflow tract (RVOT) ventricualr tachycardia (VT)

Background In patients with RVOT VT; CARTO mapping permits ablation guided by a VT complex, which may facilitate ablation of VT cases. However, the mapping system may be geometrydependent, and it has not been validated in the unique geometry of the RVOT.

Methods 30 patients with left bundle branch block and right axis VT, no history of structurally cardiac disease and normal left ventricular function underwent CARTO guided ablation.

Results The procedure was acutely successful in 27 of 30 patients, 3 had failed ablation. During a mean follow-up of 6 months, 26 of 30 patients remained arrhythmia-free.

Conclusions In this study, CARTO mapping was safely and effectively used to guide ablation of patients with RVOT VT.

\section{e0558 EFFECTS OF RIGHT VENTRICULAR APICAL PACING ON CARDIOPULMONARY FUNCTION IN PATIENTS WITH NORMAL HEART FUNCTIONS}

doi:10.1136/hrt.2010.208967.558

Xie Ying, Zhang Yuan, Liu Shuwang, Gao Wei. Peking University Third Hospital

Objective To estimate the effects of long term right ventricular apical pacing on cardiopulmonary functions in patients with normal heart function.

Method A total of 30 patient underwent dual-chamber pacemaker implantation with normal heart function (LVEF $>55 \%$, NYHA classification I-II) were enrolled and divided into two groups according to the percentage of ventricular pacing (VP), VP $\leq 45 \%$ group $(n=16)$ and $V P>45 \%$ group $(n=14)$. Patients with disease of respiratory, nervous or motor systems were excluded. Cardiopulmonary exercise test (CPET) was performed in all patients. We recorded the peak oxygen uptake $\left(\mathrm{VO}_{2}\right.$ peak), anaerobic threshold (AT), ventilatory response ( $\mathrm{VE} / \mathrm{VCO}_{2}$ slope) and other parameters during the exercise. Left ventricular ejection fraction (LVEF), left ventricular end diastolic dimension (LVEDd), left ventricular enddiastolic volume (LVEDV), systolic volume and E/A were measured using echocardiography before and after the pacemaker implantation.

Results There were no significant differences in baseline characteristics between the two groups. The meantime of enrollment after pacemaker implantation was 5.8 years. Cardiopulmonary function was significantly better in $\mathrm{VP} \leq 45 \%$ group than $\mathrm{VP}>45 \%$ group. Independent-samples t-testing showed a significantly higher $\mathrm{VO}_{2}$ peak $(19.8 \pm 3.3 \mathrm{ml} / \mathrm{kg} \cdot \mathrm{min}$ vs $17.5 \pm 2.5 \mathrm{ml} / \mathrm{kg} \cdot \mathrm{min}, \mathrm{p}=0.047)$ and AT $(18.5 \pm 1.4 \mathrm{ml} / \mathrm{kg} \cdot \mathrm{min}$ vs $16.6 \pm 2.3 \mathrm{ml} / \mathrm{kg} \cdot \mathrm{min}, \mathrm{p}=0.038)$ in $\mathrm{VP} \leq 45 \%$ group than $\mathrm{VP}>45 \%$ group. While $\mathrm{VE} / \mathrm{VCO}_{2}$ slope $(31.4 \pm 3.0$ vs $35.1 \pm 5.9, p=0.04)$ was significantly lower in $\mathrm{VP} \leq 45 \%$ group than $\mathrm{VP}>45 \%$ group. But there were no significant differences with respect to the LVEF and other echocardiography parameters between the two groups.

Conclusion Long Term right ventricular apical pacing is associated with the deterioration of cardiopulmonary function in patients with normal heart functions. Cardiopulmonary exercise test is a sensitive diagnostic method to show the early changes of cardiac function.

\section{e0559 EFFECTS OF LONG-TERM RIGHT VENTRICULAR APICAL PACING ON LEFT VENTRICULAR REMODELLING AND CARDIAC FUNCTION}

doi:10.1136/hrt.2010.208967.559

Ren Xiaoqing, Zhang Shu, Pu Jielin, Wang Fangzheng. Fuwai Heart Hospital

Objectives To investigate the impacts of long-term right ventricular apical pacing on the ventricular remodelling and cardiac functions of patients with high-grade and third-degree atrioventricular blockage with normal heart structures and cardiac functions. In addition, we provide evidences for choosing an optimal electrode implantation site.

Methods Study participants included patients who were admitted for pacemaker replacements and who revisited for examinations of implated pacemakers at outpatient. Pacemakers were implanted to treat high-grade and third-degree atrioventricular blockage. At the time of pacemaker implantation, patients had normal cardiac functions and showed no serious heart diseases or cardiac dilatation. The durations from the implantation to follow-up were more than 5 years. The pacing rate was higher than $80 \%$. Patients with a left ventricular ejection fraction (LVEF) $55 \mathrm{~mm}$ were excluded. Ventricular remodelling was defined as: increase of LVEDD by $10 \%$ and a reduction of LVEF by $25 \% 5$ years after implantation. Cardiac functions were evaluated according to the New York Heart Association (NYHA) classification.

Results A total of 82 patients with a mean age of $66.97 \pm 13.19$ years (range, 12-91 years old), including 39 male and 43 female were enrolled in this study. The average duration between two assessments was 8.7 years (104.4 months). Before pacemaker implantation, the average left atrial diameter (LA), LVEDD and LVEF were $37.0 \mathrm{~mm}, 50.23 \mathrm{~mm}$ and $64.87 \%$, respectively. After the implantation, these values were $39.39 \mathrm{~mm}(p=0.000163), 50.82 \mathrm{~mm}$ $(\mathrm{p}=0.177842)$ and $60.50 \%(\mathrm{p}=0.000104)$, respectively. 4 patients $(4.87 \%)$ had ventricular remodelling with deteriorations of cardiac function. Among them, three patients had anterior wall myocardial infarction after implantation and one had type II diabetes. Clinical heart failure symptoms were not found in the patients who did not exhibit ventricular remodelling.

Conclusion Through a long period follow-up study, we found that long-term right ventricular apical pacing in patients with normal heart structure and cardiac function generally would not cause ventricular remodelling and clinical deteriorations of cardiac function. Right ventricular apical is a safe and effective site for pacing electrode wire implantation.

\section{e0560 ROLE OF SEVERITY OF OSAS ON CRP AND LEFT ATRIAL SIZE IN PATIENTS WITH PREMATURE ATRIAL CONTRACTION}

doi:10.1136/hrt.2010.208967.560

Kaviraj Bundhoo, Dingli Xu. Nanfang Hospital

Objective Recent studies have suggested an emerging link between sleep apnoea and atrial fibrillation (AF). It has also been reported that an inflammatory process is involved in the development of atrial fibrillation. In this study we hypothesised that premature atrial contractions (PAC) might be the precursor of atrial fibrillation. 